\title{
Fallübergreifende Analysen der habituellen Dispositionen von Lehrkräften zur Unterstützung sozial benachteiligter Schüler*innen sowie milieuspezifische Einbettung der handlungsleitenden Orientierungen
}

In diesem Kapitel sollen zunächst in Anlehnung an eine sinngenetische Interpretation die Ergebnisse aus den Einzelfallanalysen zusammengeführt und miteinander verglichen werden. Tertium comparationis sind hierbei die sechs modifizierten Analysedimensionen (Abschnitt 4.1). Anschließend werden im Sinne einer soziogenetischen Interpretation die milieuspezifischen Erfahrungshintergründe der interviewten Lehrkräfte analysiert. Die sozialen Zusammenhänge der Orientierungsrahmen werden für jede Lehrperson einzeln herausgearbeitet (Abschnitt 4.2).

\subsection{Fallübergreifende Analysen}

Analog zu der Systematisierung des fallinternen Vergleichs im vorherigen Kapitel, erfolgt in diesem Teilkapitel eine fallübergreifende Analyse der habituellen Dispositionen der vier Lehrkräfte anhand der Dimensionen Konstruktion der Schüler*innen- und Elternklientel (Abschnitt 4.1.1), berufsbezogenes Selbstkonzept (Abschnitt 4.1.2), Auseinandersetzung mit Spannungsfeldern der pädagogischen Arbeit (4.1.3), Positionierung innerhalb der Schule bzw. im Kollegium (Abschnitt 4.1.4), Zuschreibung von Verantwortung für Bildungschancen (Abschnitt 4.1.5) sowie Gefahren und Fallstricke der handlungsleitenden Orientierungen (Abschnitt 4.1.6). Hierbei gilt es, gemeinsame und unterschiedliche Formen der Unterstützung sozial benachteiligter Schüler*innen herauszuarbeiten.

\subsubsection{Konstruktion der Schüler*innen- und Elternklientel}

Die befragten Lehrkräfte nehmen in den Interviews Kategorisierungen von Schüler*innengruppen vor bzw. greifen auf bestimmte Differenzkategorien zurück

S. Rutter, Sozioanalyse in der pädagogischen Arbeit, Bildung und Gesellschaft, https://doi.org/10.1007/978-3-658-32065-2_4 
und setzen sich mit Fragen zum pädagogischen Umgang mit spezifischen Herausforderungen auseinander. Die Bezugnahmen tendieren zu den Diskursfeldern Schüler*innen mit Migrationshintergrund, sozioökonomisch benachteiligte Kinder und verhaltensauffällige Schüler*innen. Grundsätzlich zeigen die Analysen, dass die Lehrkräfte in der Regel jeweils zu den Themen wechseln, in denen sie sich Handlungskompetenzen zuschreiben respektive in denen sie aus ihrer Sicht über pragmatisches Handlungswissen verfügen. So thematisiert Frau Akay vorwiegend den Umgang mit Schüler*innen mit Migrationshintergrund und unterstellt hierbei aufgrund des eigenen Migrationshintergrundes und Aufwachsens im sozialen Brennpunkt eine besondere Verbundenheit zu Schüler*innen mit Migrationshintergrund, die auf einen konjunktiven Erfahrungsraum zurückzuführen ist. Darüber hinaus schreibt sich Frau Akay aufgrund des Fach- und Methodenwissens in der Heilpädagogik und der mehrjährigen Berufserfahrung in der Kinder- und Jugendpsychiatrie besondere Kompetenzen im Umgang mit sozial und emotional auffälligen Schüler*innen zu.

Frau Antonova behandelt ähnlich wie Frau Akay schwerpunktmäßig das Thema Migration, allerdings differenziert sie nicht in gleicher Weise wie Frau Akay zwischen unterschiedlichen Migrationskontexten. Etwa grenzt Frau Akay die Gruppe der geflüchteten Schüler*innen von der Gruppe der Schüler*innen mit Migrationshintergrund ab. Relevante Unterscheidungskriterien dieser beiden Schüler*inngruppen sind aus ihrer Perspektive eigene Einwanderungserfahrungen und deutschsprachliche Kompetenzen. Spricht Frau Antonova hingegen von neu eingewanderten Schüler*innen, meint sie nicht wie Frau Akay geflüchtete Schüler*innen. Flucht wird von ihr nicht thematisiert. Vielmehr geht es Frau Antonova bei neu eingewanderten Schüler*innen um die mit der Migration einhergehende Fremdheitserfahrung aufgrund geringer oder fehlender Kenntnisse der deutschen Sprache. An einigen Stellen des Interviews verwendet Frau Antonova zudem den Begriff Schüler*innen mit Migrationshintergrund - auch hier ist das Gefühl des Fremdseins und Ausgegrenztseins aufgrund geringer oder fehlender Deutschkenntnisse in der Anfangsphase der Einwanderung im Fokus. Diese thematische Schwerpunktsetzung steht in einem engen Zusammenhang mit der persönlichen Lebensgeschichte, so beschreibt Frau Antonova eigene Fremdheitserfahrungen im Zuge ihrer Einwanderung nach Deutschland. Infolge dieser konjunktiven Erfahrungen, die Frau Antonova und die neu eingewanderten Schüler*innen aus ihrer Sicht teilen, schreibt sich Frau Antonova eine besondere Empathiefähigkeit und Vorbildfunktion für diese Kinder zu.

Im Vordergrund des Interviews mit Frau Kamper steht hingegen der Umgang mit sozioökonomisch benachteiligten Schüler*innen. Hierbei spricht sie von Schüler*innen aus „bildungsfernen Elternhäusern sozial schwachen Familien“ 
(Z. 142 f.), „,bedürftige[n] Kinder[n]“ (Z. 963) sowie Schüler*innen aus Familien, ,die Probleme haben die sich nicht so gut kümmern können“ (Z. 1398). Auch in diesem Fall ist die Relevanzsetzung des Themas nicht zufällig, sondern steht mit eigenen biografischen Erfahrungen in Verbindung. Etwa berichtet Frau Kamper, selbst aus einer „bildungsfernen Familie“ (Z. 1294) zu stammen und in bescheidenen, restriktiven Lebensverhältnissen aufgewachsen zu sein. Zudem schildert sie, dass ihre Eltern mit der Erziehung überfordert gewesen seien. Die eigenen lebensgeschichtlichen Erfahrungen gleichen aus Frau Kampers Sicht den Aufwachsensbedingungen ihrer Schüler*innen. Aufgrund dieser mutmaßlichen konjunktiven Erfahrungen nimmt Frau Kamper einen besonderen Zugang zu sozioökonomisch benachteiligten Schüler*innen und Eltern an.

Herr Jansen geht primär auf das Thema der Integration einzelner verhaltensauffälliger Schüler*innen in den Klassenverband ein. So stehen im Fokus seiner Ausführungen Schüler*innen mit sonderpädagogischem Förderbedarf sowie Schüler*innen mit Verhaltensauffälligkeiten. Im Unterschied zu Frau Akay, Frau Antonova und Frau Kamper scheint bei Herrn Jansen die Konzentration auf den Themenbereich nicht mit der eigenen Biografie einherzugehen. Vielmehr beschäftigt sich Herr Jansen im Zuge seines Berufseintritts mit dem (Selbst-)Anspruch, möglichst viele Schüler*innen zu unterstützen. Hierbei präsentiert Herr Jansen sich selbst als handlungskompetent im Umgang mit verhaltensauffälligen Schüler*innen - jedoch nicht wie bei Frau Akay, Frau Antonova und Frau Kamper aufgrund unterstellter gemeinsamer biografischer Erfahrungshintergründe, sondern infolge eines beruflichen Lern- und Entwicklungsprozesses, in dem er sich systematisch Fachwissen zum Umgang mit verhaltensauffälligen Schüler*innen aneignet.

Festzuhalten ist, dass die wirtschaftlichen, sozialen und kulturellen Rahmenbedingungen im Einzugsbereich der Schulen der interviewten Lehrkräfte deutlich voneinander abweichen. So kommen die interviewten Lehrkräfte mit Themen wie Migration und sozialer Ungleichheit in unterschiedlicher Weise und Intensität in Berührung. Frau Akay und Frau Kamper unterrichten in Schulen, in denen Schüler*innen mit Migrationshintergrund sowie sozioökonomisch benachteiligte Schüler*innen überrepräsentiert sind. Die Schule von Herrn Jansen befindet sich in einer sozialräumlich durchmischten Lage, wobei ein hoher Anteil an Schüler*innen mit Migrationshintergrund zu verzeichnen ist. Frau Antonova hingegen arbeitet an einer Schule in privilegierter sozialräumlicher Lage mit einem geringen Anteil an Schüler*innen mit Migrationshintergrund sowie sozioökonomisch benachteiligten Schüler*innen. Die Analysen zeigen, dass mit dem sozialräumlichen Kontext der Schule aufseiten der interviewten Lehrkräfte die Herausbildung handlungsleitender Orientierungen und Klientelbilder einhergeht. So können in 
der Untersuchung verschiedene Perspektiven herausgearbeitet werden, die Lehrkräfte bezüglich der Schüler*innen einnehmen: Frau Akay und Frau Kamper entwerfen insgesamt eine auf mehreren Ebenen außergewöhnlich hilfsbedürftige Schüler*innenschaft. Beide Lehrerinnen berichten davon, dass die Kinder sowohl im Unterricht als auch in lebenspraktischen Angelegenheiten auffallend viel pädagogische Unterstützung benötigen. Aus Frau Akays Sicht mangelt es bei Schüler*innen aus erschwerten Familienverhältnissen an familialer Unterstützung und Förderung sowie häufig an körperlicher Zuneigung und emotionaler Zuwendung durch die Eltern. Darüber hinaus scheinen Rituale, Strukturen und Regeln im Elternhaus zu fehlen. Im Zusammenhang mit Schüler*innen aus sozioökonomisch benachteiligten Familien betont Frau Kamper vor allem die finanzielle Bedürftigkeit und Defizite in grundlegenden Lebensbereichen wie Körperpflege und Kleidung. Im Gegensatz dazu konstruiert Frau Antonova eine besonders schulleistungsstarke und schulbildungseifrige Schüler*innenschaft. Hierbei stellt sie insbesondere die enorme Leistungsfähigkeit und die intrinsische Motivation zu lernen heraus. In der kontrastiven Analyse zu Frau Akay, Frau Antonova und Frau Kamper fällt auf, dass Herr Jansen kaum die schulischen Leistungen bzw. die Schüler*innenschaft als Ganzes thematisiert, sondern vor allem die Fragestellung behandelt, inwiefern Herausforderungen, die mit Verhaltensauffälligkeiten in Verbindung stehen, in der pädagogischen Arbeit zu bewältigen sind bzw. inwiefern allgemeinbildende Schulen unter derzeitigen Rahmenbedingungen auf die Unterstützungsbedürfnisse dieser Schüler*innen eingehen (können). Aufgrund der soziokulturell „bunt gemischt[en]“ (Z. 657) Schüler*innenschaft scheint es auch ein breites Spektrum an Schulleistungen zu geben. So beschreibt Herr Jansen, dass er sich nicht nur für die leistungsschwächeren, sondern auch für die leistungsstärkeren Schüler*innen Zeit nimmt und diese herausfordert. Im Vergleich dazu kommen Frau Akay und Frau Kamper während des gesamten Interviews nicht auf leistungsstärkere Schüler*innen zu sprechen.

Die Sichtweisen auf die Schüler*innen- und Elternschaft entfalten darüber hinaus ihre Wirkung im Hinblick auf verschiedenste Aspekte des Umgangs mit den Schüler*innen und scheinen diesen maßgeblich zu strukturieren. Bei Frau Akay und Frau Kamper stehen Praktiken des Ausgleichs der angenommenen sozioökonomischen Benachteiligungslage im Mittelpunkt der pädagogischen Arbeit, beispielsweise die Einführung von Strukturen und Ritualen in der Schule sowie die materielle Unterstützung und alltagspraktische Beratung der Eltern. Im Gegensatz zu Frau Akay und Frau Kamper berichtet Frau Anotonova, dass sie ihren Unterricht abwechslungsreich gestaltet und kontinuierlich neue Lernanstöße gibt, sodass die Schüler*innen ihr Potenzial optimal entfalten können. Herr Jansen setzt sich im Sinne des (Selbst-)Anspruchs von weitestgehender Unterstützung 
aller Schüler*innen aktiv für die Integration von verhaltensauffälligen Schüler*innen bzw. von vermeintlichen Problemschüler*innen ein. Bemerkenswert ist in diesem Zusammenhang, dass das Thema Lehren und Lernen bei den interviewten Lehrkräften ungleich zur Sprache kommt. Bei Frau Antonova spielen die Unterrichtsgestaltung bzw. die Gestaltung schulischer Lernprozesse eine zentrale Rolle im Interview. So beschreibt sie ausführlich ihr Anliegen, den Kindern einen möglichst anregungsreichen, interessanten und motivierenden Unterricht zu bieten. Die Schüler*innen sollen aus ihrer Sicht Spaß haben und gerne zur Schule kommen. Darüber hinaus scheint das intrinsisch motivierte Arbeiten und gelingende Lernen Frau Antonova mit Stolz und Freude zu erfüllen und eine Bestätigung für ihre pädagogische Arbeit zu sein. Im Vergleich dazu unterstreicht Herr Jansen, dass es ihm wichtig ist, allen Kindern gerecht zu werden und niemanden zu vernachlässigen. So unterstütze er nicht nur leistungsschwächere Kinder und Schüler*innen mit sonderpädagogischem Förderbedarf bzw. Verhaltensauffälligkeiten, sondern fördere darüber hinaus gezielt leistungsstärkere Schüler*innen. Im Kontext des Themas schulische Verantwortung für die Kompensation vermuteter familialer Probleme betont Herr Jansen ferner, dass auch die Behandlung von Unterrichtsinhalten nicht zu kurz kommen darf. Inwiefern Frau Akay und Frau Kamper einen interessanten, anspruchsvollen Unterricht gestalten, der für Freude und Motivation und eine optimale Entfaltung des individuellen Leistungspotenzials sorgt, bleibt hingegen offen. Die problemorientierte Sichtweise auf die Schüler*innenschaft legt nahe, dass die beiden Lehrerinnen vorrangig ihre pädagogische Arbeit an der Kompensation von scheinbarer sozioökonomischer Benachteiligung ausrichten. So beziehen sich Frau Akay und Frau Kamper vor allem auf die allägliche Auseinandersetzung mit den vermeintlichen Defiziten ihrer Schüler*innen. Die schulische Leistungsvermittlung, die Kompetenzorientierung sowie die kognitive Aktivierung bleiben bei beiden Lehrerinnen unerwähnt bzw. scheinen die Erwartungen an die Lern- und Leistungsfähigkeit der Schüler*innen eher gering zu sein.

Ergänzend soll Erwähnung finden, dass auch bei ähnlicher Umgebungslage unterschiedliche Wahrnehmungen und Bezugnahmen auf die Schüler*innenschaft rekonstruiert werden können. So bezeichnet Frau Akay den Stadtteil, in dem sich ihre Schule befindet, als sozialen Brennpunkt und nimmt hierbei Bezug auf einen hohen Anteil an Schüler*innen mit Migrationshintergrund und Fluchterfahrung. Frau Kamper charakterisiert ihre Schule ebenfalls als Brennpunktschule, bezieht sich hierbei jedoch auf Kinder aus schulbildungsfernen Elternhäusern und sozioökonomisch benachteiligten Familien. Vor dem Hintergrund, dass soziale Ungleichheit sowie Bildungsungleichheit mehrdimensional zu betrachten sind, ist bemerkenswert, dass sich Frau Kamper im Zusammenhang einer sogenannten 
Brennpunktschule nicht mit dem Themenfeld Migration auseinandersetzt. Auffallend ist zudem, dass sowohl Frau Akay als auch Frau Kamper den Begriff sozialer Brennpunkt bzw. Brennpunktschule nicht näher ausführen. Dies deutet auf den kommunikativ-generalisierenden Wissensbestand der ,Brennpunkt'-Metapher hin.

\subsubsection{Berufsbezogenes Selbstkonzept}

Im beruflichen Selbstverständnis kommt zum Ausdruck, wie die befragten Lehrkräfte sich selbst, ihr Handeln und damit einhergehend die Schüler*innenschaft begreifen. Die Analysen zeigen, dass sich die befragten Lehrkräfte mit den schulspezifischen Voraussetzungen auseinandersetzen, ihnen Bedeutung zuschreiben und sich mit eigenen Erfahrungen einbringen. Hierbei verweisen die vorgenommenen Rekonstruktionen darauf, dass für das berufliche Selbstbild eigene biografische Erfahrungen wichtige, aber nicht ausschließliche Bestandteile sind, aus denen die befragten Lehrkräfte spezifische Kompetenzen für den Umgang mit der von ihnen konstruierten Klientel ableiten. So stellen Frau Akay und Frau Antonova in der pädagogischen Arbeit mit Schüler*innen mit Migrationshintergrund einen konjunktiven Erfahrungsraum zu diesen Kindern her. Hierbei verengen die beiden Lehrerinnen die scheinbar kollektiven biografischen Erfahrungen auf möglichst gleichartige Herkunftsbedingungen: bei Frau Akay anhand der Merkmale Aufwachsen im sozialen Brennpunkt und türkischer Migrationshintergrund, bei Frau Antonova anhand fehlender deutscher Sprachkenntnisse und Erfahrungen von sozialer Ausgrenzung zu Beginn der Einwanderung. Der eigene Migrationshintergrund ist bei beiden Lehrerinnen darüber hinaus nicht alleiniger Bestandteil des beruflichen Selbstkonzepts. So verweist Frau Akay dezidiert auf ihre Qualifikationen als Heilpädagogin, die sie im Vergleich zu Kolleg*innen mit mehr Handlungskompetenz im Umgang mit verhaltensauffälligen Schüler*innen ausstatten. Frau Antonova präsentiert sich selbst vor allem als ,Wissensvermittlerin' und beschreibt eine abwechslungsreiche und kognitiv aktivierende Unterrichtsgestaltung.

Wenngleich Frau Antonova und Frau Akay hinsichtlich ihres Einwanderungskontextes deutlich voneinander abweichen - Frau Akay stammt aus einer türkeistämmigen Gastarbeiter*innen-Familie und ist in Deutschland geboren und aufgewachsen, Frau Antonova hingegen ist im Grundschulalter von Russland nach Deutschland migriert - weisen sie in ihrem beruflichen Selbstverständnis ähnliche handlungsleitende Orientierungen in Bezug auf den Umgang mit Schüler*innen und Eltern mit Migrationshintergrund auf. Aufgrund angenommener gemeinsamer lebensgeschichtlicher Erfahrungen schreiben beide Lehrerinnen sich selbst 
einen besonderen Zugang zu Schüler*innen mit Migrationshintergrund zu. Aus ihrer Perspektive führen die scheinbar homologen biografischen Erfahrungen zu einer besonderen Empathie und einem besonderen Vertrauensverhältnis zu diesen Schüler*innen. Darüber hinaus nehmen Frau Akay und Frau Antonova eigenhändig eine Vorbildfunktion für Kinder mit Migrationshintergrund ein. Neben diesen genannten übereinstimmenden handlungsleitenden Orientierungen bestehen aber auch Unterschiede in der Sichtweise und im Umgang mit Schüler*innen mit Migrationshintergrund. Etwa nimmt Frau Akay die migrationsbedingte Vielfalt an ihrer Schule als Potenzial für alle Schüler*innen wahr und misst ihr einen bedeutsamen Stellenwert für die pädagogische Arbeit bei. Auf gesamtschulischer Ebene scheint es Bemühungen zu geben, das schulische Wohlbefinden von Schüler*innen mit Migrationshintergrund zu steigern. Zur Konstruktion des berufsbezogenen Selbstkonzepts und damit auch zur Selbstpositionierung im schulischen Kontext nimmt sie eine eindeutige Relevanzsetzung des Migrationshintergrundes vor. Den eigenen Migrationshintergrund begreift sie als bedeutsame Differenzlinie zu Kolleg*innen ohne Migrationshintergrund. Unterschiede zwischen Lehrkräften ohne Migrationshintergrund und ihr bestehen aus ihrer Sicht bezüglich der besonderen Empathie für Schüler*innen mit Migrationshintergrund sowie der vertrauensvollen Beziehung zu Schüler*innen und Eltern mit Migrationshintergrund. Frau Antonova hingegen ist bemüht und fokussiert, durch eine strukturierte Deutschsprachförderung die schulischen Chancen einer neu eingewanderten Schülerin zu erhöhen. Ihr Engagement für die polnische Schülerin beruht auf Eigeninitiative. Die fehlende kollegiale Unterstützung nimmt sie vor dem Hintergrund von wenig Berufserfahrung als Belastung wahr. Zudem fällt in der kontrastiven Analyse auf, dass die Relevanzsetzung des eigenen Migrationshintergrundes zur Konstruktion des beruflichen Selbstkonzepts und damit auch zur Positionierung innerhalb der Schule bzw. des Kollegiums durch Ambivalenz gekennzeichnet ist und je nach spezifischer Situation unterschiedlich von Frau Antonova aufgelöst wird. Zwar benennt sie ebenfalls Vorteile in der Zusammenarbeit mit Eltern mit Migrationshintergrund und schreibt sich selbst besondere Empathie und eine Vorbildfunktion für Kinder aus Einwandererfamilien zu, der Migrationshintergrund tritt hierbei aber nicht wie bei Frau Akay als bedeutsame Differenzlinie zu Kolleg*innen ohne Migrationshintergrund hervor. Darüber hinaus erscheint das ,Preisgeben“ der eigenen Migrationserfahrung eine strategische Entscheidung: Um Vertrauen zu schaffen setzt Frau Antonova in Elterngesprächen gezielt die Information ein, selbst einen Migrationshintergrund zu haben.

Frau Kamper nimmt in der Darstellung ihrer pädagogischen Arbeit hauptsächlich Bezug auf sozioökonomisch benachteiligte Schüler*innen. Hierbei schreibt sie sich selbst eine besondere Empathiefähigkeit für Familien, ,die Probleme 
haben sich nicht so gut kümmern können“ (Z. 1398) sowie ein "Gespür“ (Z. 1399) für den Umgang mit diesen Kindern und Eltern zu. Ihr eigener, nicht geradlinig verlaufender schulischer Werdegang führt aus ihrer Sicht des Weiteren zu mehr Verständnis dafür, dass Lernbiografien dieser Kinder nicht immer positiv verlaufen, sondern von Brüchen gekennzeichnet sein können. Überdies hebt Frau Kamper eine im Vergleich zu Kolleg*innen außergewöhnlich offene, vertrauensvolle Beziehung zu Kindern und Eltern in von ihr wahrgenommen problematischen Lebenslagen hervor. In der Gesamtschau weisen die unternommenen Analysen zu Frau Kampers beruflichem Selbstkonzept ähnliche Orientierungsmuster auf, die auch bei Frau Akay und Frau Antonova in Bezug auf Schüler*innen mit Migrationshintergrund rekonstruiert werden können. So führen vermeintlich homologe lebensgeschichtliche Erfahrungen zu einem besonderen Zugang zu Schüler*innen, die sie als ähnlich zu sich selbst zu erkennen glaubt. Die konjunktiven Erfahrungen bestehen in diesem Fall aus schulischen Krisen und benachteiligten Lebensverhältnissen. Eine weitere Gemeinsamkeit mit Frau Akay ist, dass Frau Kamper die Rolle als Vertraute als Alleinstellungsmerkmal im Kollegium markiert und sowohl bedürftige Schüler*innen als auch Eltern auf sie zukommen und ihren Rat suchen.

Herr Jansen bezieht sich im Interview maßgeblich auf das Thema Umgang mit verhaltensauffälligen Schüler*innen. Hierbei bewegen sich die Bezugnahmen an der Schnittstelle zu der Differenzlinie sonderpädagogischer Förderbedarf. Den Umgang mit verhaltensauffälligen Schüler*innen markiert er als einen Lernund Entwicklungsprozess, in dem sich Lehrkräfte systematisch diagnostische Kompetenzen und Fachwissen zu Ursachen und Symptomen von Verhaltensauffälligkeiten aneignen müssen. Empathie stellt aus seiner Sicht hierbei die Schlüsselkompetenzen für die pädagogische Arbeit dar. Darüber hinaus gestaltet Herr Jansen aktiv die Zusammenarbeit mit externen Fachkräften und Institutionen sowie schulintern mit der Schulleitung, Kolleg*innen, sonderpädagogischen Lehrkräften sowie Eltern. Im Gegensatz zu Frau Akay, Frau Antonova und Frau Kamper gibt es keinen Hinweis darauf, dass das Engagement von Herrn Jansen seinen Ursprung in homologen biografischen Erfahrungen hat. So ist beispielsweise die Perspektivenübernahme der Kinder nicht auf einen emotionalen Prozess, d. h. auf das vermeintliche Selbsterleben der Emotionen der Kinder wie bei Frau Akay, Frau Antonova und Frau Kamper beschränkt, sondern wird mit einer professionellen Haltung begründet.

Gemeinsam ist allen vier Fällen, dass die befragten Lehrkräfte gezielt versuchen, eine gute Beziehung zu den Schüler*innen herzustellen, die sich durch Empathie, Nähe und Vertrauen auszeichnet. Im Hinblick auf Schüler*innen mit Migrationshintergrund gelingt dies aus der Perspektive von Frau Akay und 
Frau Antonova aufgrund unterstellter gemeinsamer biografischer Erfahrungen von selbst: Qua ihrer eigenen Einwanderungsgeschichte verfügen sie über einen besonderen Zugang zu Schüler*innen und Eltern mit Migrationshintergrund und können infolgedessen eine vertrauensvolle Beziehung aufbauen. Darüber hinaus kann eine förder- und ressourcenorientierte Haltung der beiden interviewten Lehrerinnen hinsichtlich Schüler*innen mit Migrationshintergrund herausgearbeitet werden. Frau Akay und Frau Antonova bemühen sich überdies, das schulische Wohlbefinden dieser Schüler*innen zu steigern, indem sie die sprachliche Vielfalt anerkennen und wertschätzen. Eine ähnliche Orientierung zeichnet sich bei Frau Kamper im Hinblick auf Schüler*innen in scheinbar schwierigen Lebenslagen ab: Auf Basis einer konstatierten gemeinsamen Problembetroffenheit und angenommener ähnlicher biografischer Erfahrungen kann sie eine vertrauensvolle Lehrer*innen-Schüler*innen-Beziehung aufbauen und die speziellen Problemlagen sensibler deuten und bearbeiten. Frau Kamper misst dem Wohlergehen der Kinder einen großen Stellenwert bei und übernimmt Verantwortung dafür, dass es den Kindern im Klassenverband gut geht und dass von dieser Seite her günstige Voraussetzungen geschaffen werden, damit erfolgreich gelernt werden kann. Die Kinder werden von ihr nicht nur in der Rolle als Schüler*innen wahrgenommen, sondern die familialen Probleme und damit verbundene schulische Krisen rücken ebenfalls in den Blick. Herr Jansen scheint generell dafür zu sorgen, dass in der Klasse ein angemessenes Arbeitsklima herrscht, dass Konflikte für alle Beteiligten zufriedenstellend gelöst werden und die Kinder möglichst effizient lernen können. So hebt er insbesondere hervor, dass es ihm gelungen ist, sogenannte Problemschüler*innen wie das scheinbar psychisch kranke Mädchen und den Jungen mit ADHS in den Klassenverbund zu integrieren.

\subsubsection{Auseinandersetzung mit Spannungsfeldern der pädagogischen Arbeit}

Die befragten Lehrkräfte setzten sich in den Interviews mit divergierenden Handlungsanforderungen auf unterschiedlichen Ebenen auseinander, die sie als bedeutsam erachten und die nicht zugleich oder nicht in gleicher Intensität realisiert werden können. Der Umgang mit gegensätzlichen Handlungsanforderungen erfolgt hierbei von Lehrkraft zu Lehrkraft unterschiedlich. So thematisiert Frau Akay im Kontext der Darstellung, dass Kinder aus erschwerten Familienverhältnissen ein gesteigertes Bedürfnis nach Aufmerksamkeit und Zuneigung aufweisen, die Relevanz von der Herstellung einer Balance von Nähe und Distanz in LehrendenLernenden-Beziehungen. Aus ihrer Sicht ist es wichtig, professionelle Distanz 
und Grenzen in der Gestaltung der Lehrer*innen-Schüler*innen-Beziehungen zu wahren.

Frau Antonova hingegen problematisiert im Zusammenhang mit dem besonderen Verständnis für die herausfordernde Situation neu eingewanderter Kinder, wie die unzureichende schulische Deutschsprachförderung und die mangelnde Zusammenarbeit bzw. Unterstützung im Kollegium ein Bildungsrisiko für ein kürzlich eingewandertes polnisches Mädchen darstellt. Vor dem Hintergrund eines eigenverantwortlichen Umgangs mit beruflichen Anforderungen und individuellen Ressourcen im Zuge des Berufseintritts steht auf der einen Seite die Bereitschaft von Frau Antonova, das Mädchen in ihren deutschsprachlichen Kompetenzen zu fördern, und auf der anderen Seite die wahrgenommene eingeschränkte Möglichkeit, den Anspruch auch umzusetzen. In dieser konkreten Situation setzt Frau Antonova die Priorität auf die Leitung ihrer eigenen Klasse als neue berufliche Anforderung und übergibt die Deutschförderstunde wieder an die zuständige Klassenleitung, die aus ihrer Sicht das Mädchen nicht angemessen unterstützt.

Im Kontext des Themas Umgang mit und Zuständigkeit für Kinder in problematischen Lebenslagen unterscheidet Frau Kamper zwischen ,ignoranten " und , aufopferungsvollen' Lehrkräften, wobei sie sich offenkundig zu den ,aufopferungsvollen' Lehrkräften zählt. Problematisch sind aus ihrer Sicht fehlende formale Richtlinien für die Förderung bedürftiger Schüler*innen, insbesondere das Fehlen von festgelegten Grenzen des schulischen Erziehungsauftrages. So besteht aus ihrer Perspektive die Möglichkeit, dass sich ,ignorante' Lehrkräfte entziehen (können) und sich allein die ,aufopferungsvollen' Lehrkräfte mit den Spannungsfeldern und Unsicherheiten von Handlungsberechtigung, -bedarf und -druck auseinandersetzen und die Verantwortung für diese Kinder übernehmen.

Im Gegensatz zu Frau Akay, bei der es um ein Spannungsfeld auf Mikroebene geht, die widersprüchlichen Handlungsanforderungen also unmittelbar Interaktionen zwischen Lehrkräften und Schüler*innen betreffen, geht es bei Frau Antonova und Frau Kamper eher um Spannungsfelder auf Mesoebene, das heißt um die formelle Organisation der Schule, die auch Aufgaben-, Funktions- und Verantwortungsbereiche von Lehrkräften umfasst. Herr Jansen beschäftigt sich im Rahmen des Spannungsfelds von sonderpädagogischer Förderung und inklusiver Bildung auf einer Makroebene mit den scheinbar widersprüchlichen Handlungsanforderungen. So skizziert Herr Jansen am Beispiel eines vermutlich autistischen Schülers, dass inklusiver Unterricht unter derzeitigen Rahmenbedingungen an allgemeinbildenden Schulen keine angemessene Förderung für alle Kinder ermöglicht und vielmehr zu Diskriminierung führt. Ein zentraler Kritikpunkt bezieht sich auf den bürokratischen Entscheidungsprozess über den sonderpädagogischen Förderbedarf sowie auf die bildungspolitischen Vorgaben der Umsetzung. 


\subsubsection{Positionierung innerhalb der Schule bzw. im Kollegium}

Tendenziell scheint das berufliche Selbstkonzept in den Darstellungen der interviewten Lehrkräfte passgenau zu den wahrgenommenen schulspezifischen Anforderungen und Voraussetzungen. Besonders deutlich wird dies im Fall von Frau Akay und Frau Kamper, die die eigenen Stärken und Ressourcen im Umgang mit der für die jeweilige Schule ,charakteristischen' Schüler*innenschaft betonen. Frau Akay zufolge sind Schüler*innen mit Migrationshintergrund und Fluchterfahrung an der Schule überrepräsentiert, darüber hinaus weisen aus ihrer Sicht „die meisten Kinder“ (Z. 1270) Auffälligkeiten im emotionalen und sozialen Bereich auf. Aufgrund ihres eigenen Migrationshintergrundes und Aufwachsens im sozialen Brennpunkt sowie infolge des Fach- und Methodenwissens in der Heilpädagogik und der mehrjährigen Berufserfahrung in der Kinder- und Jugendpsychiatrie schreibt sich Frau Akay besondere Handlungskompetenzen im Umgang mit den besonderen Bedürfnissen dieser Schüler*innen zu. Eine ähnliche Orientierung zeigt sich bei Frau Kamper: Ihrer Einschätzung nach sind überdurchschnittlich viele Kinder aus „bildungsfernen Elternhäusern sozial schwachen Familien“ (Z. 142 f.) an ihrer Schule vertreten. Begründet durch ihre eigenen erschwerten Aufwachsensbedingungen fühlt sich Frau Kamper verbunden mit Kindern in scheinbar problematischen Lebenslagen und beteuert, besonders empathisch mit ihnen umgehen zu können.

Die Analysen verweisen darüber hinaus auf sehr unterschiedliche Einschätzungen im Kollegium, welche Schüler*innen als ,Problemfall` bzw. als auffällig gelten. Die Kategorisierungsprozesse und Zuordnungspraktiken können hierbei als Machtkämpfe im schulischen Feld interpretiert werden. So bestehen zwischen Frau Kamper und ihrer Kollegin im Kontext der vermeintlich ungepflegten Schülerin erhebliche Meinungsunterschiede darüber, ob das äußere Erscheinungsbild der Schülerin „,nicht so schlimm“ (Z. 335 f.) oder „,wirklich verwahrlost“ (Z. 339) ist. Die Formulierung der Kollegin, die Schülerin sei , wirklich verwahrlost' gleicht hierbei einer Tatsachenbehauptung, wohingegen Frau Kampers Wortwahl ,nicht so schlimm‘ Raum für einen argumentativen Austausch lässt. Die Kollegin scheint konkrete Vorstellungen von Normen und Normabweichungen in schulischen Zusammenhängen zu haben und setzt ihre Normalitätsvorstellungen im Folgenden als Maßstab für die pädagogische Arbeit, indem sie Frau Kamper auffordert, den vermeintlichen Missstand zu bearbeiten (,da musst du der Mutter mal Bescheid sagen“, Z. 341 f.). Ein Aushandlungsprozess zwischen den beiden Lehrerinnen über die Definition von Verwahrlosung und über das weitere Vorgehen findet anscheinend nicht statt bzw. thematisiert Frau Kamper nicht. Durch 
das bestimmte Auftreten und die klare Vorgabe stellt die Kollegin eine Asymmetrie zwischen sich und Frau Kamper her. Damit weist die Kollegin Frau Kamper eine unterlegene Position zu. Die Auseinandersetzungen im Zusammenhang mit der scheinbar psychisch kranken Schülerin deuten ebenfalls auf einen schulischen Machtkampf hin. So setzt sich Herr Jansen trotz der Forderung des Schulverweises seitens der Elternschaft sehr deutlich für den Verbleib der Schülerin ein und bezieht überdies Stellung gegenüber der Schulleitung. Den Konflikt löst Herr Jansen strategisch auf, indem er die Elternvertretung überzeugt, gegen die Ausgrenzung der Schülerin zu stimmen, ohne hierbei sein eigenes Wahlverhalten offen zu legen. Die Darstellungen verweisen insgesamt auf eine selbstbewusste Platzierung innerhalb des Kollegiums. Sein zielgerichtetes und selbstbestimmtes Handeln trägt zu der Konstruktion eines gleichwertigen Verhältnisses zu seinen berufserfahrenen Kolleg*innen und dem Vorgesetzten bei.

Des Weiteren ist der Eintritt in den Lehrberuf mit großen Herausforderungen verbunden. Beispielsweise kann eine große Belastung dadurch entstehen, dass es keine klaren Standards für die pädagogische Arbeit gibt, an denen sich die berufseinsteigenden Lehrkräfte orientieren können. Bei Frau Kamper führt der fehlende Referenzrahmen für den Umgang mit hilfsbedürftigen Schüler*innen und der unklare Erziehungsauftrag aus ihrer Sicht dazu, dass sich ,ignorante" Lehrkräfte entziehen (können) und sich allein die , aufopferungsvollen' Lehrkräfte mit der Unsicherheit von Handlungsberechtigung, -bedarf und -druck auseinandersetzen und die Verantwortung für diese Kinder übernehmen. Es deutet sich bereits an, dass Frau Kamper als , aufopferungsvolle' Lehrerin unzufrieden mit der Situation und auf der Suche nach Orientierung ist. Am Beispiel der kürzlich eingewanderten polnischen Schülerin zeigt sich, dass Frau Antonova vor dem Hintergrund geringer Berufserfahrung und der neuen beruflichen Anforderungen wie die eigenständige Klassenführung die Deutschförderstunde für die Schülerin als Überlastung wahrnimmt. Infolgedessen reduziert Frau Antonova ihren pädagogischen Anspruch, eine qualitativ hochwertige Deutschförderstunde anzubieten und übergibt die Deutschförderstunde entgegen ihrer Vorstellung von optimaler Unterstützung neu eingewanderter Schüler*innen wieder der Klassenleitung.

In diesem Zusammenhang ist auf die Rolle der Zusammenarbeit mit den Kolleg*innen für die Förderung sozial benachteiligter bzw. bislang im Bildungssystem ausgegrenzter Schüler*innen hinzuweisen. So berichten Frau Kamper und Frau Antonova beide mehr oder weniger enttäuscht von einem Mangel an Kooperation und Unterstützung seitens der Kolleg*innen. Das pädagogische Handeln beider Lehrerinnen scheint vor allem von persönlichem Engagement und 
Eigeninitiative gekennzeichnet und weniger in teamorientierten Maßnahmen eingebunden zu sein. Dadurch besteht die Gefahr, dass mit der Zeit gegebenenfalls das Engagement für die Unterstützung der Kinder abnimmt.

\subsubsection{Zuschreibung von Verantwortung für Bildungschancen}

Im Allgemeinen nehmen die interviewten Lehrkräfte wahr, dass Chancengleichheit im Bildungssystem nur eingeschränkt gegeben ist. Die Ursachen für die geringeren Bildungschancen einiger Schüler*innen verorten die befragten Lehrkräfte tendenziell in der Familie. Eltern spielen aus Sicht der befragten Lehrkräfte eine entscheidende Rolle für den erfolgreichen Verlauf der Bildungsbiografie der Kinder. So ist Frau Akay der Meinung, dass sich , letztendlich zu Hause das Meiste dreht" (vgl. Z. 1536). Ihr zufolge werden in der Familie die Grundstrukturen der Erziehung gelegt, die sie auch als ,starkes Fundament“ (Z. 1534) bezeichnet. An diese grundlegenden sozialisatorischen Voraussetzungen könne die Schule anknüpfen und auf ihnen aufbauen. Bei vielen Schüler*innen denkt sie, ,,wenn die aus anderen familiären Verhältnissen kämen hätten die viel mehr Chancen“ (Z. 1542 f.). Den maximal zu erreichenden Schulabschluss stellt aus ihrer Sicht die Fachhochschulreife dar - und auch das nur für einige wenige Schüler*innen. Das Erlangen der allgemeinen Hochschulreife hält sie für ihre Schüler*innenklientel für nahezu ausgeschlossen. Für die meisten Schüler*innen scheint das Äußerste, was sie erreichen können, überhaupt ein Schulabschluss und eine anschließende Ausbildung zu sein. Im Zusammenhang von Schüler*innen aus erschwerten Familienverhältnissen problematisiert Frau Akay darüber hinaus die unzureichende schulbildungsbezogene Unterstützung und Förderung durch die Eltern sowie das Fehlen von Regeln und Tagesstrukturen in der Familie. Eine ähnliche Orientierung kann bei Frau Kamper hinsichtlich Schüler*innen aus „bildungsfernen Elternhäusern sozial schwachen Familien“ (Z. 142 f.) herausgearbeitet werden. Ebenso wie Frau Akay stellt Frau Kamper die starke Bedürftigkeit der Schüler*innen ,in allen möglichen Lebensbereichen“ (Z. 143) heraus. Ihrer Ansicht nach besteht nicht nur in finanzieller Hinsicht Unterstützungsbedarf, sondern die Hilfsbedürftigkeit bezieht sich auch auf grundlegende Lebensbereiche wie Körperpflege und Kleidung. Bessere Chancen für diese Kinder versprechen sich Frau Akay und Frau Kamper vor allem durch die kostenfreie Bereitstellung von Unterrichtsmaterialien, die Einführung von Strukturen, Ritualen und Regeln in der Schule sowie die alltagspraktische Beratung der Eltern. An späterer Stelle im Interview erläutert Frau Kamper ferner, dass „,bestimmte Lebensumstände“ (Z. 1401) dazu führen können, dass Schüler*innen Auffälligkeiten und schulische 
Krisen aufweisen, die sich wiederum auf die Bildungserfolgsaussichten auswirken. Ähnlich argumentiert Herr Jansen im Kontext des an ADHS erkrankten Schülers, der aufgrund dramatischer Lebensereignisse (Trennung der Eltern mit anschließendem Tötungsversuch durch die Mutter) schulische Einbrüche erlitt. Aus seiner Sicht sei der Schüler „vielleicht mal ein Problemkind“ (Z. 882 ff.) gewesen, mittlerweile aber nicht mehr, denn die Familienverhältnisse haben sich wieder stabilisiert und dem Schüler gehe „es so ganz gut“ (Z. 881 f.). Weiterhin betont Frau Antonova in Bezug auf Schüler*innen mit Migrationshintergrund über das gesamte Interviewmaterial hinweg die Bedeutsamkeit der Integrationsbemühungen eingewanderter Eltern für die Bildungschancen der Schüler*innen. Aus ihrer Perspektive können die Eltern „Berge versetzen“ (Z. 296) und seien der „Schlüssel zum Ganzen“ (Z. 301 f.).

Grundsätzlich besteht die Gemeinsamkeit der Fälle darin, dass aus der Perspektive der Lehrkräfte die sozioökonomische Benachteiligungslage der Familien bzw. schwierige familiale Verhältnisse einen negativen Einfluss auf den schulischen Alltag, das Schulleistungsniveau und den Bildungsverlauf der Kinder haben. Insgesamt können die Darstellungen der befragten Lehrkräfte auch als diskursive Entlastungsstrategie für schulische Misserfolge gedeutet werden. So dienen die negativen Stereotype über die Erziehungsfähigkeit der Eltern beziehungsweise die Relevanzsetzung des elterlichen Engagements in dieser Perspektive als externale Kausalattribuierung für eine ungleiche Chancenverteilung, die durch pädagogische Arbeit nur begrenzt ausgeglichen werden kann.

Die Analysen zeigen darüber hinaus, dass die Lehrkräfte auch institutionelle Rahmenbedingungen als ursächlich für ungleiche Bildungschancen ansehen. Etwa illustriert Herr Jansen am Beispiel eines offensichtlich autistischen Schülers, dass unter den derzeitigen Voraussetzungen an allgemeinbildenden Schulen das gemeinsame Lernen aller Kinder "nichts mehr mit Chancengleichheit zu tun“ (Z. 1658 f.) habe, sondern zu Diskriminierung führe. In diesem speziellen Fall sei der Schüler besser an einer Förderschule aufgehoben. Geeignete Fördermöglichkeiten stellen in dieser Perspektive kleinere Klassen bei gleichzeitig mehr (speziell ausgebildetem) Lehrpersonal dar. Bezüglich der Strukturen und Möglichkeiten an allgemeinbildenden Schulen scheint Herr Jansen die offizielle Feststellung des sonderpädagogischen Förderbedarfs im Hinblick auf die Bildungschancen der Schüler*innen zu befürworten, da dadurch die Rechtsgrundlagen für eine individuelle, an den spezifischen Bedürfnissen ausgerichtete Förderung geschaffen ist. Dass es sich bei der Feststellung des sonderpädagogischen Förderbedarfs oftmals um einen Verwaltungsakt handelt, bei dem rechtliche Vorgaben über dem Einzelfall stehen, kritisiert Herr Jansen. Unter diese Perspektive lässt sich ebenfalls Frau Kampers Einschätzung fassen, dass aufgrund der enormen Hilfsbedürftigkeit 
der Schüler*innen in unterschiedlichen Lebensbereichen mehr personelle Unterstützung in den Bereichen Schulsozialarbeit und sonderpädagogische Förderung notwendig sei. Ausreichend Personalressourcen in verschiedenen pädagogischen Handlungsfeldern stellen aus ihrer Sicht Möglichkeiten dar, um der sozialen Benachteiligungslage der Schüler*innen entgegenzuwirken. In dem thematischen Zusammenhang von Aufgaben-, Funktions- und Verantwortungsbereichen der Lehrkräfte ist Frau Antonovas Kritik an der unzureichenden Deutschförderung der neu eingewanderten polnischen Schülerin durch die zuständige Klassenleitung einzuordnen, die die schulischen Erfolgschancen des Mädchens verringere. In Übereinstimmung mit Frau Akay stellt eine strukturierte Deutschförderung eine geeignete Möglichkeit dar, Schüler*innen mit geringen oder keinen Deutschkenntnissen institutionell zu unterstützen und schulische Integration zu erleichtern.

Die Vorstellungen der befragten Lehrkräfte darüber, wie sie selbst einen Beitrag zur Bearbeitung von Bildungsungleichheit leisten können und sozial benachteiligte und bisher im Bildungssystem ausgegrenzte Schüler*innen am besten fördern können, scheinen im Grundsatz ähnlich zu sein. Wie bereits im Kontext des beruflichen Selbstverständnisses erläutert, sind die interviewten Lehrkräfte bemüht, atmosphärische Voraussetzungen in der Klasse zu schaffen, die dafür sorgen, dass sich die Schüler*innen wohl und aufgehoben fühlen und optimal lernen können. Dazu gehört vor allem die Gestaltung eines vertrauensvollen Verhältnisses zu den Schüler*innen, wobei jede Lehrperson eine eigene Art und Weise hat, auf die Schüler*innen zuzugehen. Etwa besprechen Frau Akay und Frau Antonova bewusst ihre eigene Einwanderungsgeschichte mit den Schüler*innen mit Migrationshintergrund und signalisieren ihnen dadurch, dass sie nahe an ihrer Lebenswelt sind und die scheinbar gleichen Erfahrungen teilen. Dadurch können sie die eigene Vorbildrolle authentisch gestalten. Ähnlich hierzu unterstreicht Frau Kamper ihr Bemühen, sich Kindern aus vermeintlich problematischen Familienverhältnissen auch von einer sehr privaten Seite zu zeigen und ihnen aufgrund ihrer eigenen Biografie viel Verständnis für familiale Probleme entgegenzubringen. Herr Jansen hingegen versucht den Kindern bewusst zu zeigen, dass er deren Perspektiven nachvollziehen kann. Zu den Sozialkompetenzen von Lehrkräften zählt aus seiner Sicht die Fähigkeit, sich ,in die Lage des Gegenübers hineinzuversetzen“ (Z. 1958 f.). Seine berufliche Leitlinie ist, „die Welt mit Kinderaugen sehen“ (Z. 1958). Dazu gehört für ihn, die Ursachen für auffälliges Verhalten zu ermitteln, wie er am Beispiel einer hörgeschädigten Schülerin illustriert. Prinzipiell wird über das gesamte Interviewmaterial deutlich, dass sich Frau Akay, Frau Antonova und Frau Kamper in besonderem Maße für die Schüler*innen verantwortlich fühlen, denen sie ähnliche biografische Erfahrungshintergründe zuschreiben, und entsprechend förderorientiert handeln. 
Im Gegensatz dazu kann bei Herrn Jansen die besondere Selbstverpflichtung für verhaltensauffällige Schüler*innnen rekonstruiert werden. Die Verantwortungsübernahme für gerade diese Schüler*innen stützt sich aber im Gegensatz zu Frau Akay, Frau Antonova und Frau Kamper nicht auf eine vermeintlich homologe Lebensgeschichte, sondern steht im Zusammenhang mit einer lernenden Schule und der Gestaltung der eigenen beruflichen Rolle.

\subsubsection{Gefahren und Fallstricke der handlungsleitenden Orientierungen}

In den Fallbeschreibungen von Frau Akay, Frau Antonova und Frau Kamper wurde bereits die Konstruktion eines konjunktiven Erfahrungsraumes kritisch beleuchtet. So führen die beiden Lehrerinnen mit Migrationshintergrund einen besonderen Zugang und eine große Nähe zu Schüler*innen mit Migrationshintergrund auf gemeinsame (Differenz-)Erfahrungen im Zuge der Migration bzw. auf die Zugehörigkeit zur selben national-ethnischen Gruppe sowie auf gleiche Sozialisationsbedingungen zurück. Entsprechende Sinn- und Bedeutungszuschreibungen, die in einem angenommenen konjunktiven Erfahrungsraum begründet sind, können auch bei Frau Kamper rekonstruiert werden. Sie stellt die besondere Vertrauensbasis zu sozial benachteiligten Schüler*innen über vermeintlich ähnliche schulbildungsbiografische Erfahrungen und einem gleichartigen familialen Hintergrund her. Mit der Vorstellung eines konjunktiven Erfahrungsraumes geht jedoch die Gefahr einher, dass die eigenen biografischen Erfahrungen unreflektiert auf die Schüler*innen übertragen werden und damit die Besonderheit des Falles aus dem Blick gerät. Dies kann zur Folge haben, dass auf die Bedürfnisse der Schüler*innen abgestimmte Handlungsperspektiven nicht entworfen werden. Ein weiteres Risiko besteht darin, dass die Lehrkräfte möglicherweise nur die benachteiligten und bisher im Bildungssystem ausgegrenzten Schüler*innen bevorzugen, mit denen sie sich über vermeintlich ähnliche lebensgeschichtliche Erfahrungen verbunden fühlen. Eine solche ,biografische Befangenheit ${ }^{\star}$ zeichnet sich bereits bei den befragten Lehrerinnen ab. Etwa fühlt sich Frau Akay insbesondere mit Schüler*innen verbunden, die eine ähnliche national-ethnische Zugehörigkeit aufweisen. Zu Schüler*innen mit Fluchterfahrung scheint sie beispielsweise in keiner weiteren Beziehung zu stehen. Zumindest thematisiert sie bis auf die fehlenden deutschen Sprachkenntnisse zu Beginn des Schuleintritts keine anderen mit der Flucht in Verbindung stehenden Herausforderungen wie z. B. psychische Belastungen und Traumatisierungen. Damit vergleichbar ist Frau Antonovas Sichtweise auf das neu eingewanderte polnische Mädchen. So bleibt 
die unterstellte Gemeinsamkeit auf die Fremdheitserfahrung im Zuge der Migration beschränkt, ungleiche familiale Ressourcen und Sozialisationsbedingungen zwischen ihr und der Schülerin sowie verschiedene Ausgangslagen in divergierenden Heimatländern lässt sie außer Acht. Darüber hinaus ist bedeutsam, dass sie zu den Kindern eine Affinität aufweist, die ähnlich leistungsorientiert sind wie sie selbst. Im Fall von Frau Kamper scheint der Fokus der pädagogischen Arbeit ebenfalls auf Schüler*innen zu liegen, die der eigenen Herkunft besonders ähnlich erscheinen. So beruht die habituelle Übereinstimmung auf scheinbar homologen Erfahrungen von Vernachlässigung durch die Eltern, restriktiven Lebensumständen und schulischen Brüchen. An der Unterstützung und Förderung von beispielsweise Schüler*innen mit Migrationshintergrund und Schüler*innen mit sonderpädagogischem Förderbedarf scheint sie hingegen unbeteiligt zu sein.

Die pädagogische Arbeit der drei Lehrerinnen scheint damit insgesamt besonders für die Schüler*innenklientel förderlich, die mehr Übereinstimmung mit den jeweiligen eigenen biografischen Erfahrungen und Haltungen aufweisen. Im Gegensatz zu den drei Lehrerinnen können bei Herrn Jansen keine konstatierten gemeinsamen lebensgeschichtlichen Erfahrungen zu Schüler*innen rekonstruiert werden. Jedoch steht auch bei ihm eine ganz bestimmte Schüler*innenklientel im Mittelpunkt seiner Ausführungen: Schüler*innen mit Verhaltensauffälligkeiten bzw. Schüler*innen mit sonderpädagogischem Förderbedarf. Der Bezug zu Schüler*innen, die bislang ebenfalls im Bildungssystem benachteiligt und ausgegrenzt werden, wie zum Beispiel Schüler*innen mit Migrationshintergrund, scheint zu fehlen, jedenfalls geht er im Interview auf diese Schüler*innengruppe nicht ein.

Neben diesen spezifischen ,Blindstellen' kann darüber hinaus eine defizitorientierte Sichtweise auf den familialen Hintergrund bislang im Bildungssystem benachteiligter und ausgegrenzter Schüler*innen festgestellt werden. Frau Akay und Frau Kamper zufolge scheint vor allem ein niedriger sozio-ökonomischer Status ein Hindernis für gelingende Sozialisations- und (Schul-)Bildungsprozesse darzustellen. Etwa problematisieren die beiden Lehrerinnen, dass die große Bedürftigkeit in mehreren Lebensbereichen sowie der Mangel an angemessener elterlicher Unterstützung bei Schulangelegenheiten die pädagogische Arbeit erschwert und zu ungleichen Bildungschancen führt. Die defizitorientierte Perspektive kann hierbei den vielfältigen Bemühungen der befragten Lehrkräfte, den Bildungserfolg der Schüler*innen zu unterstützen, im Wege stehen. So zeichnen sich bei Frau Akay und Frau Kamper geringe Erwartungen an die Lernfähigkeit der Schüler*innen ab, die sich auch in einer entsprechenden Unterrichtsgestaltung mit einem eher (zu) geringen Anforderungsniveau niederschlagen (können). Aus diesem Blickwinkel besteht die Gefahr, dass Frau Akay und Frau Kamper primäre Herkunftseffekte verstärken. Vor dem Hintergrund, dass die Eltern 
als maßgebender Faktor für den erfolgreichen Verlauf der Bildungsbiografie der Kinder angesehen werden, erscheinen die Defizitzuschreibungen an die elterliche Bildung- und Erziehungsfähigkeit und die negativen Stereotype über den sozioökonomischen Status besonders brisant, da sie ein Hindernis für eine gelingende Zusammenarbeit zwischen Eltern und Lehrkräften bzw. Schule darstellen können. So lässt die von Frau Kamper geschilderte Auseinandersetzung mit einer Mutter darauf schließen, dass Frau Kampers wohlgemeinte Ratschläge bezüglich angemessener Kleidung seitens der Mutter als Bevormundung und Übergriff verstanden werden. Die problembezogene Kommunikation kann zur Folge haben, dass sich die Kooperation zwischen der Mutter und Frau Kamper perspektivisch schwierig gestaltet. In diesem Kontext ist auch auf die negativen Wertungen von Schüler*innen und Eltern und die Grenzziehung zwischen Lehrkräften und Eltern im Fall von Herrn Jansen hinzuweisen. Gleichwohl er eine möglichst konfliktfreie Zusammenarbeit mit den Eltern im Interesse einer optimalen Entwicklung der Kinder anstrebt, ist fraglich, inwiefern die Kommunikation mit den Eltern durch Respekt und Wertschätzung seinerseits gekennzeichnet ist. Generell scheinen die Eltern in keinem ebenbürtigen Verhältnis zu den befragten Lehrkräften zu stehen bzw. es scheint keine Kommunikation auf Augenhöhe stattzufinden. Naheliegend ist, dass Eltern sich infolgedessen von der Schule distanzieren.

\subsection{Milieuspezifische Differenzierungen}

Ähnlich wie in Kapitel 3 soll im Folgenden dem Einzelfall Rechnung getragen werden, sodass für jede der vier Lehrpersonen die milieuspezifischen Erfahrungshintergründe, die den handlungsleitenden Orientierungen zugrunde liegen, analysiert werden. So thematisieren die befragten Lehrkräfte zum Teil selbst während des Interviews die eigene Sozialisations- und Lebensgeschichte und schreiben ihr unterschiedliche Bedeutung für die pädagogische Arbeit zu. Zum Teil wurden die Lehrkräfte auch durch die Interviewerin zur Auseinandersetzung mit der Frage nach dem Einfluss biografischer Erfahrungen auf das berufliche Handeln angeregt. Da die Wahrnehmungs-, Denk- und Handlungsschemata vorreflexiv und den Lehrkräften in der Regel nicht bewusst zugänglich sind, konnten sie aus den Erzählungen und Beschreibungen nur indirekt erschlossen werden. Das heißt, die explizit genannten lebensgeschichtlichen Erfahrungen, die die befragten Lehrkräfte als bedeutsam für die pädagogische Arbeit markieren, geben zwar Aufschluss über den Erfahrungsraum der handlungsleitenden Orientierungen, nicht aber über die Entstehungsgeschichte der herausgearbeiteten 
Orientierungsrahmen. Für die Analyse der Milieuspezifität der handlungsleitenden Orientierungen werden im Zuge dieses Interpretationsschrittes nicht nur die bereits dargestellten Passagen, sondern das gesamte Interview mit einbezogen, um soziale Zusammenhänge zwischen unterschiedlichen Orientierungsrahmen systematisch herausarbeiten zu können. Zur Schärfung der Analyse des Einzelfalls von Frau Akay und Frau Antonova wird in diesem Kapitel auf die bereits vorgestellte Milieustudie „Migration - Teilhabe - Milieus“ von (Geiling, Gardemin, Meise, \& König, 2011) Bezug genommen. Die milieuspezifische Differenzierung von Frau Kamper und Herrn Jansen erfolgt hingegen auf Grundlage des Milieukonzepts von Vester, von Oertzen, Geiling, Hermann und Müller (2001).

\subsubsection{Frau Akay - türkische Bildungsaufsteigerin mit großer sozialer Sensibilität}

Frau Akay bezeichnet sich selbst als „Gastarbeiterkind“ (Z. 1023) und verweist auf ihre „türkische Herkunft“ (Z. 935). Ferner sei sie in Deutschland geboren und in einem sozial benachteiligten Stadtteil aufgewachsen. Unter Berücksichtigung ihres Alters zum Interviewzeitpunkt muss die arbeitsmarktbezogene Einwanderung ihrer Eltern von der Türkei nach Deutschland demnach vor den 1980er Jahren stattgefunden haben. Im Anschluss an das Interview berichtet Frau Akay, dass sie in ihrer eigenen Schulzeit ungerecht behandelt wurde: Trotz guter Schulleistungen in der Grundschule erhielt sie eine Hauptschulempfehlung und wurde als leistungsstarke Schülerin von den Lehrkräften an der Hauptschule nicht dabei unterstützt, auf eine höhere Schulform zu wechseln. Frau Akay kritisiert, dass sich ihre Eltern damals nicht gegen die Schullaufbahnempfehlung und institutionellen ,Haltekräfte“ zur Wehr setzten. So beendete sie auch ihre Schulzeit mit dem Hauptschulabschluss und begann danach eine Ausbildung im medizinischen Bereich. Nach Abschluss der Ausbildung nahm sie an der Fachhochschule ein Studium der Heilpädagogik auf und arbeitete darauffolgend mehrere Jahre in der Kinder- und Jugendpsychiatrie, bevor sie sich erfolgreich auf die Stellenanzeige der Schule als Förderschullehrerin bewarb.

Die Ausführungen zum familialen Hintergrund und der Schulzeit dokumentieren zunächst, dass Frau Akay eine Reihe von Erfahrungen der Ethnisierung und Differenz gemacht hat. Beispielsweise spricht sie nicht von einem benachteiligten Stadtteil, sondern von einem ,sozialen Brennpunkt“ (Z. 916). In diesem Zusammenhang ist auch die Identitätskonstruktion als türkisches „Gastarbeiterkind“ (Z. 1023) bedeutsam. Der Begriff ,Gastarbeiter*in“ bezeichnet die seit Mitte der 1950er Jahre gezielt im Ausland angeworbenen Arbeitskräfte, die zeitlich befristet 
den Arbeitskräftemangel in Deutschland kompensieren sollten. Den türkeistämmigen Gastarbeiter*innen wurden hierbei explizit un- und angelernte Positionen im deutschen Berufssystem zugewiesen, für die keine deutschen Arbeitskräfte zu finden waren. Das niedrige Einkommensniveau und die vielfachen Diskriminierungserfahrungen in Deutschland führten dazu, dass sich türkeistämmige Arbeitnehmer*innen von Beginn an in segregierten Stadtteilen niederließen bzw. in diese verwiesen wurden (Geiling, Gardemin, Meise, \& König, 2011). Insgesamt zeigt sich, dass mit ,der damit verbundenen sozialen Unterschichtung der ersten Generation der Türkeistämmigen in Deutschland [...] ein noch in den Folgegenerationen wirksamer Nachteil in der Konkurrenz um soziale Positionen und um das dafür notwendige Bildungskapital aus[geht]“ (ebd., S. 272 f.). Der Befund zur systematischen Schlechterstellung türkeistämmiger Gastarbeiter*innen und deren Nachkommen spiegelt sich auch in den Aussagen von Frau Akay wider: Neben den benachteiligten Aufwachsensbedingungen im sozialen Brennpunkt erlebte sie vor allem eine Ungleichbehandlung während der Schulzeit, indem ihr als leistungsstarke Schülerin eine höhere Schulformempfehlung verwehrt und sie an der Hauptschule trotz guter Schulleistungen , gehalten“ wurde. Die Kritik an die mangelnde Unterstützung durch die Eltern verweist auf deren Unsicherheit im Umgang mit pädagogischen Autoritäten. $\mathrm{Zu}$ Bedenken ist auch, dass geringe Deutschkenntnisse der Eltern bzw. fehlende Vertrautheit mit dem deutschen Bildungssystem ursächlich für deren Zurückhaltung gewesen sein könnten. Frau Akay scheint demnach aus einem sozial eher benachteiligten Herkunftsmilieu zu stammen.

Anhand der Schilderungen zum familialen Hintergrund sowie des schulischen und beruflichen Werdegangs wird darüber hinaus ersichtlich, dass Frau Akay für sich eine soziale Aufstiegsbiografie konstruiert, obgleich anhand der Aussage, sie sei ein ,Gastarbeiterkind" und in einem ,sozialen Brennpunkt" aufgewachsen, keine gesicherten Rückschlüsse auf die Beweggründe der Migration, der früheren Lebenssituation, dem Bildungsstand und den Berufen der Eltern gezogen werden können. Dennoch stellen ihre Schilderungen einen sukzessiven, vertikalen Aufstiegsprozess aus den unterschichteten sozialen Positionen ihrer Eltern dar, den sie scheinbar aus eigener Kraft und ohne erkennbare bzw. wahrgenommener familialer Unterstützung verwirklichte.

Neben den scheinbar eigenmotivierten Bildungsanstrengungen sowie den Umwegen bis zum akademisierten Beruf lassen sich einige Hinweise finden, die auf das Milieu der bildungsorientierten Aufsteiger*innen (ebd.) schließen lassen. Zum einen dokumentiert sich an Frau Akays Bildungsbiografie eine Orientierung an höherer Bildung: Nach dem Hauptschulbesuch folgte eine Ausbildung im medizinischen Bereich, anschließend studierte sie erfolgreich 
Heilpädagogik an der Fachhochschule. Typischerweise sind bildungsorientierte Aufsteiger*innen zum Großteil in pädagogischen und sozialen beruflichen Handlungsfeldern beschäftigt. Mit der Berufsfindung ist zum anderen die große soziale Sensibilität als wesentlicher Zug der Bildungsorientierten verknüpft, die auf der eigenen sozialen Benachteiligungslage beruht. Zugleich finden die Erfahrungen der Ethnisierung und Differenz Eingang in das berufliche Selbstkonzept, wobei Angehörige dieses Milieus den eigenen Erfahrungshintergrund nutzen, um anderen Einwander*innen als Vorbild zu dienen (ebd.). Etwa eignet sich Frau Akay das Label ,Migrationshintergrund " und ,Aufwachsen im sozialen Brennpunkt" als Ressource für ihre pädagogische Arbeit an und markiert Vorteile sowohl für Kinder mit Migrationshintergrund als auch für Kinder ohne Migrationshintergrund sowie positive Effekte für Kolleg*innen ohne Migrationshintergrund. Schließlich sei noch auf die Grenzen des Aufstiegs hingewiesen. Den bildungsorientierten Aufsteiger*innen ist der Weg in Elitepositionen der Gesellschaft bzw. der Zugang zu gesellschaftlich führenden sozialen Milieus bislang noch versperrt und auch das akademische Feld hat sich ihnen nicht ohne Weiteres geöffnet (ebd.). Bei Frau Akay entsteht der Eindruck, dass sie für ihren sozialen Aufstieg stärker kämpfen musste und die Realisierung ihr ,nur' durch einen zeitintensiveren Step-by-stepProzess gelang. Ihr Bildungsaufstieg schien mit großer Mühe und zum Teil auch mit Rückschlägen (z. B. die nicht den guten Schulleistungen entsprechende Hauptschulempfehlung) verbunden zu sein.

\subsubsection{Frau Antonova - Bildungswiederaufsteigerin aus der ehemaligen Sowjetunion mit starker Leistungsorientierung}

Frau Antonova erzählt im Anschluss an das Interview, dass sie im Alter von zehn Jahren gemeinsam mit ihrer Mutter und ihrem älteren Bruder von der ehemaligen Sowjetunion nach Deutschland migriert sei. Die Einwanderung erfolgte demnach Mitte der 1990er Jahre. Ihr Vater blieb in Russland, da sich ihre Eltern kurz vor der Migration trennten. Darüber hinaus berichtet Frau Antonova, dass ihre Mutter in Russland studiert habe. Frau Antonova bezeichnet sich selbst indirekt als sogenannte (Spät-)Aussiedlerin bzw. scheint sich dieser Gruppe prinzipiell zugehörig zu fühlen. Im Hinblick auf ihre eigene Schulzeit nimmt Frau Antonova eine ungerechte Behandlung wahr, so bekam sie aufgrund der Schulnote ,befriedigend im Fachunterricht Deutsch statt einer Gymnasial- eine Realschulempfehlung. Die damals bindende Schullaufbahnempfehlung konnte aus ihrer Sicht nur durch das 
große Engagement der Mutter und das Wohlwollen eines Schulleiters am Gymnasium umgangen werden (,,da hat sich aber meine Mutter dann damals äh stark für eingesetzt ist dann ähm zum Gymnasium gegangen und hat mich dann-hat der Schulleiter hat das dann auch zugelassen und zwar sollte ich keine Gymnasiumempfehlung kriegen weil ich eine Drei in Deutsch ja und dann ähm (.) hat aber der Schulleiter des Gymnasiums gesagt das wäre kein Problem“, Z. 25 ff.). Nach dem Abschluss der Schulzeit mit der Hochschulreife absolvierte Frau Antonova zunächst eine Ausbildung zur Erzieherin und studierte im Anschluss Lehramt.

Im Allgemeinen verweisen die Ausführungen zum biografischen Hintergrund auf ein eher schulbildungsnahes und privilegiertes Herkunftsmilieu. So betont Frau Antonova den akademischen Hintergrund der Mutter und beschreibt diese als engagiert und widerstandsfähig gegenüber pädagogischen Autoritäten. Das proaktive Handeln der Mutter kann als Kampf gedeutet werden, das in dem Herkunftsland erworbene (höhere) Bildungskapital gegen eine Entwertung im Zuge der Migration zu verteidigen. Die Auswanderung als alleinerziehende Mutter von zwei Kindern kann zudem als Hinweis auf einen selbstsicheren Umgang mit Herausforderungen interpretiert werden.

Wichtig in dem Zusammenhang der Analyse der Milieuspezifität erscheint, dass im deutschen Migrationsdiskurs den (Spät-)Aussiedler*innen als deutsche Minderheiten eine besondere Rolle zukommt. So sind (Spät-)Aussiedler*innen aus Ostmitteleuropa, Ost- und Südosteuropa sowie der ehemaligen Sowjetunion als im Zweiten Weltkrieg vertriebene deutsche ,Volkzugehörige' den deutschen Staatsangehörigen gleichgestellt und haben einen rechtlichen Anspruch darauf, in Deutschland aufgenommen zu werden. In der Bundesrepublik bekommen sie in der Regel automatisch die sogenannte ,Statusdeutscheneigenschaft". Aufgrund der deutschen Herkunft und der speziellen Einwanderungsprozedur nach Deutschland, im Rahmen derer das Bekenntnis zum deutschen Volkstum verlangt wird, identifizieren sich viele (Spät-)Aussiedler*innen bereits vor ihrer Migration als Deutsche (Geiling, Gardemin, Meise, \& König, 2011). Diese historischen, rechtlichen und politischen Aspekte erscheinen konstitutiv für Frau Antonovas Identitätskonstruktion. So ist zum einen Frau Antonovas Bestreben, sich anzupassen und als Einwanderin möglichst wenig aufzufallen, sehr ausgeprägt. In diesem Kontext könnte auch die situations- und kontextspezifische Relevanzsetzung des eigenen Migrationshintergrundes gedeutet werden: Grundsätzlich vermeidet Frau Antonova eine Adressierung als „Migrationsandere“ (Mecheril, 2010, S. 17) - die Ausnahme besteht darin, wenn es aus ihrer Sicht dem schulischen Vorankommen der Kinder mit Migrationshintergrund dient. Zum anderen konnte ein assimilatorisches Integrationsverständnis herausgearbeitet werden. Hierbei präsentiert Frau Antonova im Modus einer, Etablierten-Außenseiter-Figuration“ (Elias \& 
Scotson, 1993) ihre Einwanderungsgeschichte als idealtypische Integration. Zentrales Unterscheidungsmerkmal zwischen den , etablierten Integrierten ' und den , abseits der Mehrheitsbevölkerung stehenden Integrationsunwilligen` stellt aus ihrem Blickwinkel die individuelle Anstrengung dar. Die von der Mehrheitsbevölkerung eingeforderte Anpassung und Integrationsleistung wird demnach von Frau Antonova fraglos akzeptiert und tradiert. Die Betonung der Anpassungsund Leistungsbereitschaft der Mutter sowie die durchaus prätentiöse Darstellung der eigenen Person können ferner eine Strategie im Umgang mit Erfahrungen der Ethnisierung und Differenz darstellen bzw. als Ausdruck des Ringens um soziale Anerkennung verstanden werden (Geiling, Gardemin, Meise, \& König, 2011, S. 167).

Die starke Orientierung an individueller Leistung und sowie die Anpassungsbereitschaft an bestehende Hierarchien sind überdies Anzeichen für das eher konservative Milieu der erfolgsorientierten Aufsteiger*innen (ebd.). Der Leistungsindividualismus stellt hierbei für die (Spät-)Aussiedler*innen eine angemessene Strategie dar, ihre soziale Position $\mathrm{zu}$ verbessern oder $\mathrm{zu}$ erhalten. Etwa verfügt Frau Antonovas Mutter offenbar trotz Abwertung des mitgebrachten Bildungskapitals infolge der Migration noch über ausreichend Ressourcen, um ihre Tochter vor der nicht den Schulleistungen entsprechenden Realschulempfehlung, die einem sozialen Abstieg gleicht, zu schützen. Die Mutter bewältigt den schnellen Wiederaufstieg hierbei anscheinend mit Disziplin und leistungsindividualistischen Einstellungen (ebd.). Ferner bildet die eigene Anpassung- und Leistungsbereitschaft, bzw. die der Mutter, den Mittelpunkt von Frau Antonovas gesellschaftspolitischem Anspruch und ist für sie der Schlüssel zur sozialen Integration. Die Leistungsorientierung findet darüber hinaus Ausdruck in der pädagogischen Arbeit. So hat Frau Antonova einen besonderen Sinn für leistungsstarke und dem Anstrengungs- und Leistungsprinzip folgenden Schüler*innen und gestaltet dementsprechend ihren Unterricht.

\subsubsection{Frau Kamper - Bildungsaufsteigerin aus problematischen Familienverhältnissen mit ausgeprägter Sicherheitsorientierung}

Im Kontext des beruflichen Selbstverständnisses wurde ausführlich erläutert, dass Frau Kamper ihre biografischen Erfahrungen selbstreflexiv in einem engen Zusammenhang mit ihrer Berufswahl als Grundschullehrerin sowie ihrer gezielten Entscheidung für eine sogenannte Brennpunktschule mit einer besonders unterstützungsbedürftigen Schüler*innenklientel stellt. Hierbei gewährt Frau Kamper 
einen tieferen Einblick in ihren familialen Hintergrund und die allgemeinen Lebensumstände ihrer Kindheit und Jugendphase. Etwa beschreibt sie, dass sie in bescheidenen, finanziell knappen Verhältnissen aufgewachsen sei. Ihre Herkunftsfamilie bezeichnet Frau Kamper selbst als „bildungsfern“ (Z. 1294) und erläutert hierzu, dass ihre Mutter zu Beginn keine Berufsausbildung hatte, sondern der Schwerpunkt auf der Gründung einer eigenen Familie lag. Darüber hinaus betont Frau Kamper aber auch Bildungsbemühungen der Mutter. Hinzu tritt retrospektiv ihr Eindruck von einer deutlichen Überforderung der eigenen Eltern mit der Kindererziehung, so deutet Frau Kamper an, durch ihre Eltern als Kind emotional vernachlässigt geworden zu sein. Über ihre eigene Bildungsbiografie berichtet Frau Kamper überdies, dass ihre Schulzeit von häufigen Schulwechseln sowie ständig wechselnden schulleistungsbezogenen Höheund Tiefpunkten gekennzeichnet war. Bei den schulischen Krisen konnte sie anscheinend auf keine familiale Unterstützung zurückgreifen. Insgesamt präsentiert Frau Kamper eine beschwerte Kindheit und Jugend und stellt Belastungen der Aufwachsensbedingungen wie die sozioökonomische Schlechterstellung, die emotionale Vernachlässigung durch die Eltern sowie schulleistungsbezogene Aufund Abwärtsbewegungen in den Vordergrund ihrer Darstellungen. Mit Kindern, denen sie ähnliche problematische Familienverhältnisse zuschreibt, identifiziert sie sich. Die vermeintlich homologen biografischen Erfahrungen hinsichtlich bescheidener, restriktiver Lebensumstände sowie mit der Kindererziehung überlasteter Eltern führen aus ihrer Perspektive zu einem besonderen Zugang zu diesen Schüler*innen. Die bewusste Entscheidung für ein Grundschullehramtsstudium sowie für eine sogenannte Brennpunktschule hängt bei Frau Kamper demnach stark von biografisch beeinflussten, ideellen Motiven ab.

Die Beschreibungen zum sozioökonomischen Hintergrund deuten insgesamt auf ein eher schulbildungsfernes und sozial benachteiligtes Herkunftsmilieu. Das Phänomen der elterlichen Überforderung bzw. der emotionalen Vernachlässigung wird prinzipiell in allen sozialen Milieus zu finden sein, jedoch weist die Eintrittswahrscheinlichkeit eine sozial ungleiche Verteilung auf. Darüber hinaus verfügen Angehörige sozial benachteiligter Milieus über weniger Ressourcen, um diese besonderen Belastungen zu kompensieren. Im Gegenteil: Vielmehr werden sie durch die finanzielle Knappheit sowie ungünstige Wohnbedingungen noch verstärkt (Drucks, Bauer, \& Hastaoglu, 2011). Wie im Fall von Frau Kamper können die familialen Beeinträchtigungen die Entwicklung von Schulleistungen und den Verlauf von Schulkarrieren negativ beeinflussen.

Wesentliches Thema des Interviews ist der institutionelle Umgang mit einer aus Frau Kampers Sicht außergewöhnlich hilfsbedürftigen Schüler*innenschaft. Anhand der im gesamten Interview präsenten Auseinandersetzung mit den 
pädagogischen Spannungsfeldern Handlungsbedarf, Handlungsberechtigung und Handlungsdruck wird Frau Kampers starke Sicherheitsorientierung deutlich. So wünscht sie sich einen formalen Bezugsrahmen, der Zuständigkeiten für dieser Schüler*innen regelt und einfordert, mit dem Ziel, , aufopferungsvolle ' Lehrkräfte zu entlasten. Dass geordnete Verhältnisse und Strukturen für Frau Kamper sehr wichtig sind, zeigt sich auch an der Differenzierung unterschiedlicher pädagogischer Berufsgruppen wie Förderschullehrkräfte und Schulsozialarbeiter*innen, denen sie spezifische, von ihrer Arbeit abgrenzbare Funktions- und Aufgabenbereiche zuschreibt. Unter dem Aspekt der Sicherheitsorientierung erscheint auch die Berufswahl milieuspezifisch begreiflich, denn der Lehrberuf bietet eine gehobene, aber abgesicherte berufliche Position.

Die grundlegende Erfahrung von bescheidenen und restriktiven Lebensumständen, die Bildungsbemühungen der Mutter sowie die ausgeprägte Orientierung an Sicherheit und festen Ordnungsstrukturen deuten insgesamt auf das Milieu der traditionellen kleinbürgerlichen Arbeitnehmer*innen. Grundsätzlich wird erkennbar, dass der intrinsisch motivierte Bildungsaufstieg große Mühe und Anstrengung erforderte, wobei Frau Kamper scheinbar auf keinerlei Vorbilder und Unterstützung in der Familie zurückgreifen konnte. In einer milieuspezifischen Untersuchung von Studierenden in den Sozialwissenschaften weisen Lange-Vester und Teiwes-Kügler (2006) nach, dass beim aufstiegsorientierten Typus aus der ständisch-kleinbürgerlichen Traditionslinie neben Aufstiegsambitionen auch Selbstzweifel und Unsicherheit auftreten. So kann Frau Kamper zwar auf einen beachtlichen Aufstieg durch Bildung zurückblicken, dies scheint aber nicht zu habitueller Selbstsicherheit geführt zu haben. Etwa lässt sich Frau Kamper im Kontext der Auseinandersetzung mit einer Mutter, die sich über den Eingriff in ihr Privatleben beschwert hat, in ihrer Zuständigkeit für die vermeintlichen familialen Probleme verunsichern. Grundsätzlich hat Frau Kamper durch den sozialen Bildungsaufstieg zwar ihre Position im sozialen Raum verändert, diese Wandlung scheint aber weniger die grundlegenden Orientierungen und Wertbezüge ihres Herkunftsmilieus zu betreffen.

\subsubsection{Herr Jansen - habituell selbstsicherer Lehrer aus bessergestelltem Herkunftsmilieu mit grundlegender Orientierung an Partizipation}

Auf Nachfrage der Interviewerin schildert Herr Jansen, dass es ihm im Vergleich zu den Schüler*innen, die er jetzt unterrichtet, in seiner eigenen Kindheit 
und Jugendphase „,sehr sehr gut“ (Z. 2631) ging. So habe er ,eine gute Erziehung genossen“ (Z. 2654), auf die er zurückgreifen könne. Denn obwohl er „,vielleicht auch mal ein bisschen Scheiße gemacht habe“ (Z. 2659 f.) und er „damals zwischendurch vom rechten Weg vielleicht ein bisschen abgekommen“ (Z. 2666 f.) sei, kann er sich der guten Erziehung ,immer noch bedienen und die auch (.) irgendwie abrufen“ (Z. 2671 f.). Im Anschluss an das Interview führt Herr Jansen aus, was er mit ,vielleicht auch mal ein bisschen Scheiße gemacht [zu haben]“ (Z. 269 f.) und ,zwischendurch vom rechten Weg vielleicht ein bisschen abgekommen [zu sein]“ (Z. 2666 f.) meint. Etwa sei er während seiner eigenen Schulzeit phasenweise nicht an Schule und Unterricht interessiert gewesen und habe häufiger unentschuldigt in der Schule gefehlt. Das Desinteresse und die mangelnde schulische Motivation haben dazu geführt, dass seine schulischen Leistungen stark nachließen und er in der Mittelstufe eine Klasse wiederholen musste. In der Oberstufe kam es erneut zum Leistungsabfall und Herr Jansen wechselte unter schulischem Druck das Gymnasium, auf dem er letztlich die Hochschulreife erwarb. Sein unmittelbar anschließendes Lehramtsstudium und auch der Vorbereitungsdienst verliefen nach seinen Aussagen hingegen reibungslos.

Insgesamt scheint Herr Jansen aus einem eher schulbildungsnahen und privilegierten Herkunftsmilieu zu stammen. So zeugt die Darstellung der schulischen Leistungseinbrüche und des Schulwechsels kurz vor dem Abitur von Gelassenheit und Zuversicht im Umgang mit potenziell krisenhaften schulischen Situationen, die wiederum auf familialen Ressourcen zu beruhen scheinen.

Herr Jansens Berufseintritt fällt mit der Phase der praktischen Einführung des Förderschwerpunktes Hören und Kommunikation in der Grundschule zusammen. Die neuen beruflichen Anforderungen handhabt Herr Jansen selbstsicher und selbstbestimmt: Etwa interpretiert er das gemeinsame Unterrichten von Schüler*innen mit sonderpädagogischem Förderbedarf und Schüler*innen ohne sonderpädagogischen Förderbedarf als fortlaufenden Lern- und Entwicklungsprozess einer jeden Lehrperson. Hierbei eignet sich Herr Jansen systematisch diagnostische Kompetenzen und Fachwissen zu Ursachen und Symptomen von Verhaltensauffälligkeiten an und gestaltet aktiv die interne wie externe Zusammenarbeit mit Akteuren und Institutionen. Grundsätzlich orientiert Herr Jansen sein pädagogisches Handeln an weitestgehender Unterstützung und Förderung aller Schüler*innen. Diesen Anspruch vertritt er mitunter selbstbewusst gegenüber Eltern, Kolleg*innen und Schulleitung, wie anhand der Geschehnisse um die vermeintlich psychisch kranke Schülerin deutlich wird. Derweil ist Herr Jansen darum bemüht, dass möglichst alle schulischen Akteure einvernehmlich Verantwortung auch für offensichtliche und auf unbestimmte Zeit schwierige 
Schüler*innen übernehmen. Grenzen des gemeinsamen Lernens werden aus Sicht von Herrn Jansen durch die inadäquaten Voraussetzungen an allgemeinbildenden Schulen gesteckt. Am Beispiel eines scheinbar autistischen Schülers wird deutlich, dass Herr Jansen eine bestmögliche Unterstützung und Förderung aller Schüler*innen an dem Aspekt der Machbarkeit bzw. Durchführbarkeit orientiert. In diesem Zusammenhang ist auch das kritische Bewusstsein gegenüber den bildungspolitischen und rechtlichen Vorgaben bedeutsam. So werden von Herrn Jansen gesellschaftliche Anforderungen handlungspraktisch auf den Prüfstand gestellt.

Zusammengenommen weist die Selbstsicherheit, das eigenverantwortliche und prinzipiell solidarische pädagogische Handeln sowie die aktive Entwicklung von Professionalität auf das Milieu der modernen Arbeitnehmer*innen (Geiling, Gardemin, Meise, \& König, 2011) hin. Etwa scheint Herr Jansen für sich Gestaltungsmöglichkeiten in einer lernenden Schule einzunehmen bzw. sein Schulumfeld eröffnet Gestaltungsspielraum für engagierte einzelne Lehrkräfte.

Open Access Dieses Kapitel wird unter der Creative Commons Namensnennung 4.0 International Lizenz (http://creativecommons.org/licenses/by/4.0/deed.de) veröffentlicht, welche die Nutzung, Vervielfältigung, Bearbeitung, Verbreitung und Wiedergabe in jeglichem Medium und Format erlaubt, sofern Sie den/die ursprünglichen Autor(en) und die Quelle ordnungsgemäß nennen, einen Link zur Creative Commons Lizenz beifügen und angeben, ob Änderungen vorgenommen wurden.

Die in diesem Kapitel enthaltenen Bilder und sonstiges Drittmaterial unterliegen ebenfalls der genannten Creative Commons Lizenz, sofern sich aus der Abbildungslegende nichts anderes ergibt. Sofern das betreffende Material nicht unter der genannten Creative Commons Lizenz steht und die betreffende Handlung nicht nach gesetzlichen Vorschriften erlaubt ist, ist für die oben aufgeführten Weiterverwendungen des Materials die Einwilligung des jeweiligen Rechteinhabers einzuholen.

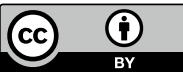

